\title{
Probing antimatter gravity - The AEGIS experiment at CERN
}

A. Kellerbauer ${ }^{1, a}$, S. Aghion ${ }^{2,3}$, C. Amsler ${ }^{4}$, A. Ariga ${ }^{4}$, T. Ariga ${ }^{4}$, G. Bonomi ${ }^{5,6}$, P. Bräunig ${ }^{7}$, J. Bremer ${ }^{8}$, R. S. Brusa ${ }^{9,10}$, L. Cabaret ${ }^{11}$, M. Caccia ${ }^{3,12}$, R. Caravita ${ }^{13,14}$, F. Castelli ${ }^{3,15}$, G. Cerchiari ${ }^{1}$, K. Chlouba $^{15}$, S. Cialdi ${ }^{3,15}$, D. Comparat ${ }^{11}$, G. Consolati ${ }^{2,3}$, A. Demetrio ${ }^{7}$, L. Di Noto ${ }^{13,14}$, M. Doser ${ }^{8}$, A. Dudarev ${ }^{8}$, A. Ereditato ${ }^{4}$, C. Evans ${ }^{2,3}$, R. Ferragut ${ }^{2,3}$, J. Fesel $^{8}$, A. Fontana ${ }^{6}$, S. Gerber ${ }^{8}$, M. Giammarchi ${ }^{3}$, A. Gligorova ${ }^{17}$, F. Guatieri9 ${ }^{9,10}$, S. Haider ${ }^{8}$, H. Holmestad ${ }^{18}$, T. Huse ${ }^{18}$, E. Jordan ${ }^{1}$, M. Kimura ${ }^{4}$, T. Koettig ${ }^{8}$, D. Krasnický14, V. Lagomarsino ${ }^{13,14}$, P. Lansonneur ${ }^{19}$, P. Lebrun $^{19}$, S. Lehner ${ }^{20}$, J. Liberadzka ${ }^{8}$, C. Malbrunot $^{8,20}$, S. Mariazzi ${ }^{20}$, V. Matveev ${ }^{21,22}$, Z. Mazzotta ${ }^{3,15}$, G. Nebbia ${ }^{23}$, P. Nédélec ${ }^{19}$, M. Oberthaler ${ }^{7}$, N. Pacifico ${ }^{17}$, D. Pagano ${ }^{5,6}$, L. Penasa ${ }^{9,10}$, V. Petráček ${ }^{16}$, C. Pistillo ${ }^{4}$, F. Prelz ${ }^{3}$, M. Prevedelli ${ }^{24}$, L. Ravelli, ${ }^{9,10}$, B. Rienäcker ${ }^{8}$, O.M. Røhne. ${ }^{18}$, A. Rotondi ${ }^{6,25}$, M. Sacerdoti ${ }^{3,15}$, H. Sandaker ${ }^{18}$, R. Santoro ${ }^{3,12}$, P. Scampoli ${ }^{4,26}$, L. Smestad ${ }^{8,27}$, F. Sorrentino ${ }^{13,14}$, M. Špaček ${ }^{16}$, J. Storey ${ }^{4}$, I.M. Strojek ${ }^{16}$, G. Testera ${ }^{14}$, I. Tietje ${ }^{8,28}$, E. Widmann ${ }^{20}$, P. Yzombard ${ }^{11}$, S. Zavatarelli ${ }^{14}$, J. Zmeskal ${ }^{20}$, and N. Zurlo6,29

(AEGIS Collaboration)

\footnotetext{
${ }^{1}$ Max-Planck-Institut für Kernphysik, Saupfercheckweg 1, 69117 Heidelberg, Germany

${ }^{2}$ Politecnico of Milano, Piazza Leonardo da Vinci 32, 20133 Milano, Italy

${ }^{3}$ INFN Milano, via Celoria 16, 20133 Milano, Italy

${ }^{4}$ Laboratory for High Energy Physics, Albert Einstein Center for Fundamental Physics, University of Bern, 3012 Bern

${ }^{5}$ Department of Mechanical and Industrial Engineering, Univ. of Brescia, via Branze 38, 25123 Brescia, Italy

${ }^{6}$ INFN Pavia, via Bassi 6, 27100 Pavia, Italy

${ }^{7}$ Kirchhoff-Institut für Physik, Univ. of Heidelberg, Im Neuenheimer Feld 227, 69120 Heidelberg, Germany

${ }^{8}$ Physics Department, CERN, 1211 Geneva 23, Switzerland

${ }^{9}$ Department of Physics, University of Trento, via Sommarive 14, 38123 Povo, Trento, Italy

${ }^{10}$ TIFPA/INFN Trento, via Sommarive 14, 38123 Povo, Trento, Italy

${ }^{11}$ Laboratoire Aimé Cotton, University Paris-Sud, 91405 Orsay Cedex, France

${ }^{12}$ Department of Science, University of Insubria, via Valleggio 11, 22100 Como, Italy

${ }^{13}$ Department of Physics, University of Genova, via Dodecaneso 33, 16146 Genova, Italy

${ }^{14}$ INFN Genova, via Dodecaneso 33, 16146 Genova, Italy

${ }^{15}$ Department of Physics, University of Milano, via Celoria 16, 20133 Milano, Italy

${ }^{16}$ Czech Technical University, Prague, Brehová 7, 11519 Prague 1, Czech Republic

${ }^{17}$ Institute of Physics and Technology, University of Bergen, Allégaten 55, 5007 Bergen, Norway

${ }^{18}$ Department of Physics, University of Oslo, Sem Sælandsvei 24, 0371 Oslo, Norway

${ }^{19}$ Institute of Nuclear Physics, CNRS/IN2p3, University of Lyon 1, 69622 Villeurbanne, France

${ }^{20}$ Stefan Meyer Institute for Subatomic Physics, Austrian Academy of Sciences, Boltzmanngasse 3, $1090 \mathrm{Vi-}$ enna, Austria

${ }^{21}$ Institute for Nuclear Research of the Russian Academy of Science, 117312 Moscow, Russia

${ }^{22}$ Joint Institute for Nuclear Research, 141980 Dubna, Russia
}

a e-mail: a.kellerbauer@cern.ch 
${ }^{23}$ INFN Padova, via Marzolo 8, 35131 Padova, Italy

${ }^{24}$ University of Bologna, viale Berti Pichat 6/2, 40126 Bologna, Italy

${ }^{25}$ Department of Physics, University of Pavia, via Bassi 6, 27100 Pavia, Italy

${ }^{26}$ Department of Physics, University of Napoli Federico II, Complesso Universitario di Monte S. Angelo, 80126 Napoli, Italy

${ }^{27}$ Research Council of Norway, Drammensveien 288, 0283 Oslo, Norway

${ }^{28}$ Physics Department, Technical University Berlin, Straße des 17. Juni 135, 10623 Berlin, Germany

${ }^{29}$ Department of Civil Engineering, University of Brescia, via Branze 43, 25123 Brescia, Italy

\begin{abstract}
The weak equivalence principle states that the motion of a body in a gravitational field is independent of its structure or composition. This postulate of general relativity has been tested to very high precision with ordinary matter, but no relevant experimental verification with antimatter has ever been carried out. The AEGIS experiment will measure the gravitational acceleration of antihydrogen to ultimately $1 \%$ precision. For this purpose, a pulsed horizontal antihydrogen beam with a velocity of several $100 \mathrm{~m} \mathrm{~s}^{-1}$ will be produced. Its vertical deflection due to gravity will be detected by a setup consisting of material gratings coupled with a position-sensitive detector, operating as a moiré deflectometer or an atom interferometer. The AEGIS experiment is installed at CERN's Antiproton Decelerator, currently the only facility in the world which produces copious amounts of low-energy antiprotons. The construction of the setup has been going on since 2010 and is nearing completion. A proof-of-principle experiment with antiprotons has demonstrated that the deflection of antiparticles by a few $\mu \mathrm{m}$ due to an external force can be detected. Technological and scientific development pertaining to specific challenges of the experiment, such as antihydrogen formation by positronium charge exchange or the position-sensitive detection of antihydrogen annihilations, is ongoing.
\end{abstract}

\title{
1 Introduction
}

The study of antimatter in the laboratory has the potential to elucidate one of the great unsolved questions in physics: Why does the observable universe appear to contain only ordinary baryonic matter and no sizable amounts of antimatter? In order to solve the baryon asymmetry riddle, several experiments at CERN's Antiproton Decelerator (AD) [1] are studying antihydrogen $(\overline{\mathrm{H}})$, the simplest atomic antimatter system. According to the CPT theorem [2], particles and antiparticles must have exactly the same properties - up to a sign. (C stands for charge conjugation, $\mathrm{P}$ for parity and $\mathrm{T}$ for time reversal.) Thus, the comparison of different properties of hydrogen and its antimatter counterpart can shed light on the question of CPT violation. As was shown almost 50 years ago for CP violation [3], also CPT violation can result in a baryon asymmetry [4]. Until today, experimental tests have provided no indication of CPT violation.

The CPT theorem has been proven to be valid under several mild assumptions, such as locality, unitarity and Lorentz invariance. In addition, the proof requires that the physical system in question be described by a quantum field theory (QFT). Gravity is notably the only one of the four fundamental interactions which is not formulated as a QFT, but rather as a geometric property of space and time. According to the weak equivalence principle (WEP) of general relativity, the motion of a test body in a gravitational field does not depend on the body's structure and composition. In a hypothetical quantum gravity theory, a variety of exchange particles with different coupling constants and ranges is possible [5]. For instance, current results of torsion balance measurements with ordinary matter allow for the existence of additional vector (spin-1) and scalar (spin-0) exchange bosons, under the condition that their coupling constants and ranges are identical [6]. 


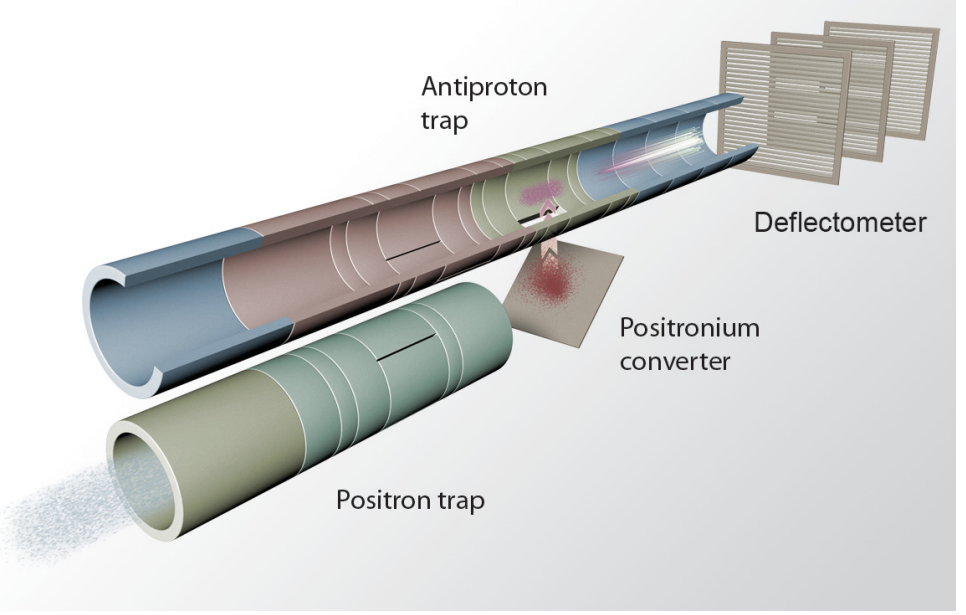

Figure 1. Three-dimensional cut-open sketch of the central part of the AEGIS apparatus, showing the low-field Penning traps, the positronium converter and the deflectometer or interferometer.

The question of an anomalous gravitational acceleration of antimatter has been the subject of considerable controversy from a theoretical point of view. Over the past decades, a number of arguments have been brought forward against it [7]. A prominent early example is Morrison's objection against anti-tensor gravity, which he showed would violate energy conservation [8]. Others are based on the observation that ordinary atoms contain virtual antiparticles due to vacuum polarization, or on the fact that certain CPT tests should be sensitive to the underlying gravitational potential. However, all of these theoretical arguments make some non-trivial assumptions, such as CPT invariance, or the somewhat arbitrary choice of an absolute gravitational potential. A comprehensive review which is relevant for a WEP test at the $1 \%$ level is given in Ref. [9]. Ultimately, a direct experimental study of antimatter gravity is required to positively resolve the issue.

While the WEP has been tested up to a relative precision of $1.8 \times 10^{-13}$ with ordinary matter [10], no relevant measurement of the gravitational acceleration of antimatter has been carried out. This is because until recently only electrically charged antimatter particles - such as positrons $\left(e^{+}\right)$ and antiprotons $(\bar{p})$ - were readily available, which are too sensitive to stray electromagnetic fields to permit a gravity measurement [11]. This situation changed in 2002, when copious amounts of cold $\overline{\mathrm{H}}$ were synthesized for the first time by the ATHENA experiment [12] installed at the AD. ATHENA created anti-atoms via the three-body reaction $\bar{p}+2 e^{+} \longrightarrow \overline{\mathrm{H}}+e^{+}$. For this purpose, $\bar{p}$ and $e^{+}$were brought into overlap in the nested electric potential of a Penning trap [13]. Today $\overline{\mathrm{H}}$ is routinely produced in large quantities by several AD experiments. Therefore, a WEP test with a neutral antimatter particle has finally come within reach.

The AEGIS experiment (Antimatter Experiment: Gravity, Interferometry, Spectroscopy) was proposed in 2007 [14, 15]. Its main goal is to measure for the first time the force acting on $\overline{\mathrm{H}}$ atoms in the gravitational field of the earth [16]. The experiment is based on the creation of a cold, bunched beam of $\overline{\mathrm{H}}$ and the measurement of its vertical displacement after a substantial horizontal flight, as shown in Fig. 1. Ultimately, the aim is to measure the acceleration $g$ acting on the anti-atoms to $1 \%$. The apparatus is centered around ion traps placed inside superconducting magnets for ion confinement and manipulation. AEGIS will use a novel $\overline{\mathrm{H}}$ production technique involving positronium (Ps), the bound state of $e^{+}$and $e^{-}$. Antihydrogen will be formed via the charge exchange reaction $\mathrm{Ps}^{*}+\bar{p} \longrightarrow \overline{\mathrm{H}}^{*}+e^{-}$, where the asterisk denotes an excited state. In combination with an efficient cooling technique for $\bar{p}$, this scheme can create ultracold $\overline{\mathrm{H}}$ for high-precision gravity measurements. 


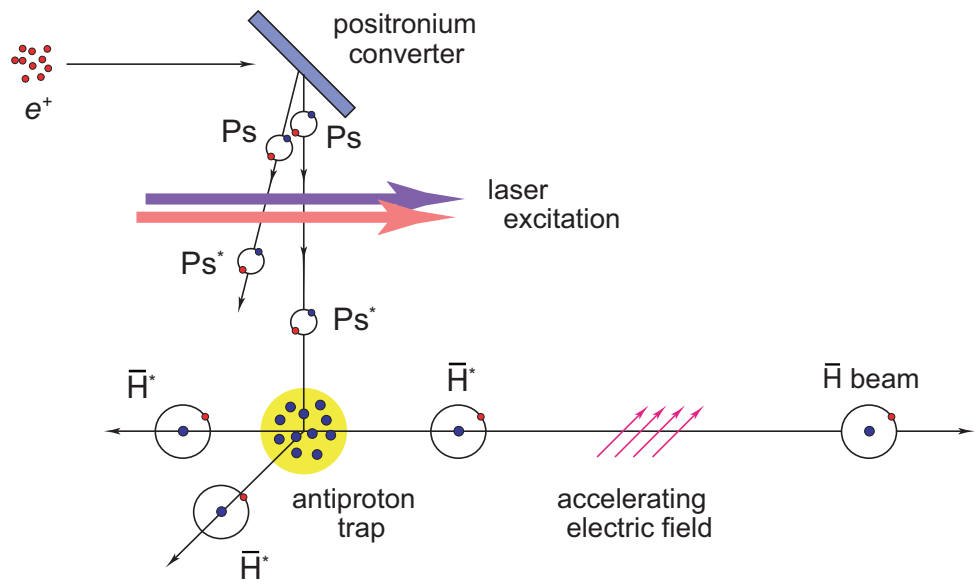

Figure 2. Scheme used in AEGIS for $\overline{\mathrm{H}}$ recombination by resonant charge exchange with positronium followed by Stark acceleration.

\section{Experiment}

The AEGIS experiment involves the following main experimental steps: (1) Capture of $\bar{p}$ from the AD in a Penning trap, followed by electron cooling; (2) Production of $e^{+}$from a source of $\beta^{+}$-decaying radioisotope; (3) Production of Ps by implanting $e^{+}$into a nanoporous converter material; (4) Excitation of Ps to a Rydberg state; (5) Recombination of $\overline{\mathrm{H}}$ by resonant charge exchange between Rydberg Ps and cold $\bar{p}$; (6) Formation of an $\overline{\mathrm{H}}$ beam by Stark acceleration with inhomogeneous electric fields; (7) Measurement of the vertical acceleration $g$ in a deflectometer or interferometer. All of the aforementioned techniques have been demonstrated independently. The extraordinary challenge of AEGIS lies in combining all of them while reconciling potentially conflicting requirements, such as a high magnetic field for ion confinement and cooling and the absence of electromagnetic fields for highprecision force measurements.

\subsection{Production of antihydrogen by resonant charge exchange}

Antihydrogen formation by three-body recombination is a well-established method that has been demonstrated to create $\overline{\mathrm{H}}$ at high rate [17]. As mentioned above, AEGIS will make use of an alternative technique which involves Ps as precursor [18]. While it necessarily results in a lower $\overline{\mathrm{H}}$ production rate, the method has a unique advantage: A plasma of $\bar{p}$ confined in the ion trap can be pre-cooled to the temperature of the surrounding cryogenic environment or even beyond. When $\overline{\mathrm{H}}$ is formed by resonant charge exchange between Ps and $\bar{p}$, the temperature of the produced anti-atoms is close to that of the $\bar{p}$ prior to the interaction. This is because the mass of $\bar{p}$ is almost 2000 times larger than that of $e^{ \pm}$, which therefore only impart a very small momentum on their reaction partners. Using a suitable $\bar{p}$ cooling method, such as sympathetic cooling by laser-cooled atomic $[19,20]$ or molecular anions [21], the technique can thus potentially create ultracold $\overline{\mathrm{H}}$.

In the AEGIS experiment, positronium will be synthesized by depositing a bunch of cold positrons into a positronium converter consisting of a nanoporous insulator or semiconductor wafer [22, 23], as shown in Fig. 2. The converter is placed a short distance away from the trap where cold $\bar{p}$ have been prepared. In the pores of the material, $e^{+}$pick off $e^{-}$from the surface and form Ps, which thermalize with the bulk by multiple scattering. Finally, cold Ps are isotropically emitted from the surface. The conversion efficiency has been shown to be as high as 30\% [24]. Before the Ps reach the $\bar{p}$ cloud where they form $\overline{\mathrm{H}}$, they are excited to a Rydberg level [25]. In this way, the cross-section of the 
(a)

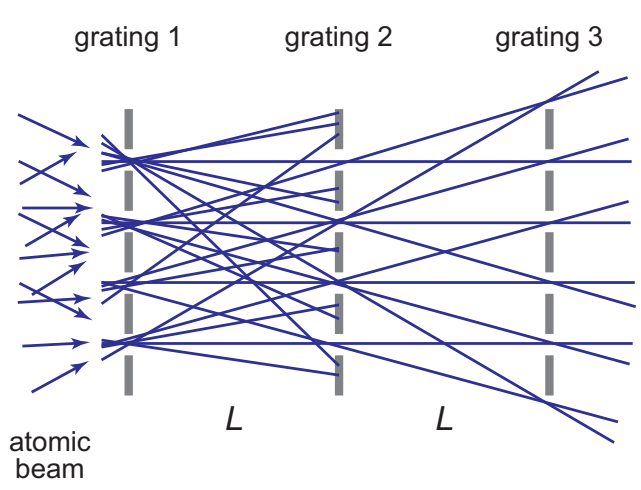

detector

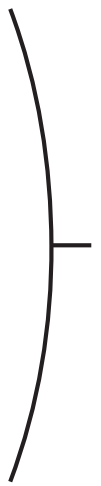

(b) relative intensity
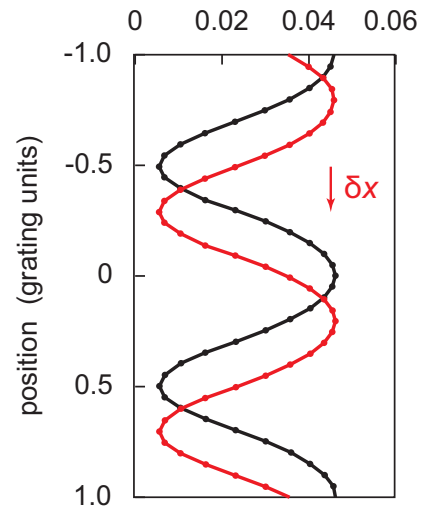

Figure 3. (a) Principle sketch of the deflectometry/interferometry technique, showing a setup with three identical gratings and a non-position-sensitive detector. (b) Monte Carlo simulation of the signal detected behind a threegrating moiré deflectometer as a function of the analysis grating's position. The red dots and solid line show the shift in the presence of a force perpendicular to the beam axis.

charge exchange reaction, which scales with the fourth power of the principal quantum number of the Ps, is significantly enhanced. Due to energy conservation, $\overline{\mathrm{H}}$ is also created in a narrow, well-defined range of Rydberg states. Finally, the $\overline{\mathrm{H}}$ cloud is formed into a beam by Stark acceleration [26].

\subsection{Principle of gravity measurement}

The force acting on a slow beam of massive particles can be measured with a series of slits or gratings. In the latter case, the device is called a moiré deflectometer or a matter wave interferometer, depending on the diffraction angle $\phi=\lambda_{\mathrm{dB}} / d$, where $\lambda_{\mathrm{dB}}$ is the particles' de Broglie wavelength and $d$ is the grating period. As shown in Fig. 3(a), two material gratings spaced a distance $L$ apart create a shadow or interference pattern a further distance $L$ downstream. A third grating with identical grating period is placed at the location where the line pattern forms. The transmission of particles as a function of the third grating's position (in the direction of the force) is measured by an appropriate detector located beyond the device. As illustrated in Fig. 3(b), the transmission maxima yield the displacement of the beam, and hence, the force acting on it. In AEGIS, an antihydrogen beam accelerated to $500 \mathrm{~m} \mathrm{~s}^{-1}$ will be deflected by about $10 \mu \mathrm{m}$ over a flight path of $1 \mathrm{~m}$.

A three-grating moiré deflectometer has been used some 20 years ago to measure the acceleration due to gravity of metastable argon atoms [27]. As an alternative to a movable third grating, a positionsensitive detector can be placed in its position. In this way, the arrival positions for particles moving at different velocities can be recorded simultaneously and without any moving parts. The current AEGIS design, which is still being finalized, calls for such a setup with a sophisticated detector that can record the annihilation position of impinging anti-atoms with a vertical resolution of about $2 \mu \mathrm{m}$. The detector will most likely consist of a combination of complementary technologies, including a nuclear photographic emulsion and a silicon strip or pixel detector. Both types of systems have been extensively tested in the AEGIS setup and found to deliver the required position resolution and detection efficiency for an antihydrogen gravity measurement [28, 29]. 


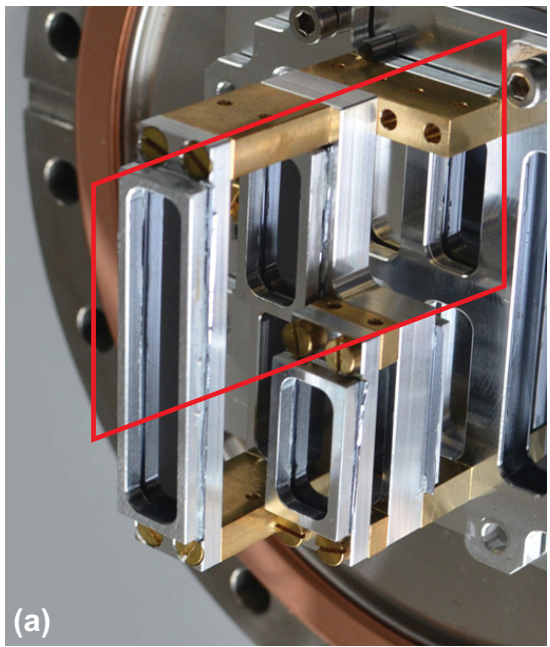

(b)
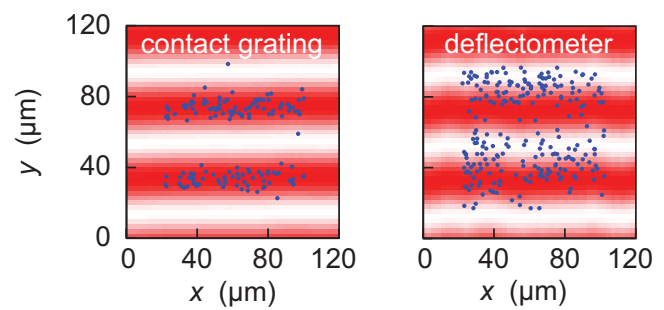

(c)

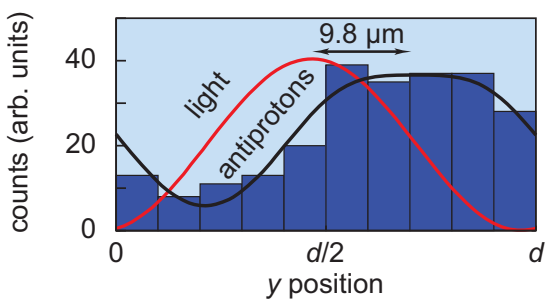

Figure 4. (a) Photograph of the miniaturized moiré deflectometer setup used in the proof-of-principle measurement with a $\bar{p}$ beam. (b) Positions of recorded $\bar{p}$ annihilation events (blue dots) and light pattern (red) behind the contact grating (left) and the deflectometer setup (right). (c) Antiproton annihilation events summed up modulo the grating period (blue bins and black line), compared to the intensity of the light pattern (red line).

\section{Recent experimental results}

The construction of the AEGIS apparatus began in 2010. As of 2015, all major components except for the gravimeter have been completed and installed in the experimental zone. The $e^{+}$source and accumulator, the two superconducting magnets and all ion traps have been constructed and commissioned. Antiproton pulses from the AD have been captured and up to $4 \times 10^{5} \bar{p}$ have been stacked in the catching trap. Storage times of close to $10 \mathrm{~min}$ were achieved. Similarly, several $10^{8} e^{+}$have been confined in the accumulator for storage times of up to $20 \mathrm{~min}$. A multi-layer scintillating-fiber tracker detector, to be installed around the central ion traps in order to monitor $\overline{\mathrm{H}}$ production, has been commissioned with a radioactive source [30]. The laser system for Ps excitation has been completed and commissioned [31]. In parallel, development work towards the gravimeter is ongoing. In the following, two recent significant advances towards an antimatter gravity measurement will be presented.

\subsection{Deflectometry with antiprotons}

In order to demonstrate the principle of a force measurement by deflectometry and to test the position resolution of the emulsion detector, we carried out a test experiment with a $\bar{p}$ beam [32]. Antiprotons are supplied directly by the $\mathrm{AD}$, and hence are available in large numbers, yet they produce a starshaped annihilation signature in the emulsion very similar to that of $\overline{\mathrm{H}}$. A miniaturized deflectometer consisting of two gratings spaced only $L=25 \mathrm{~mm}$ apart and with a grating period of $d=40 \mu \mathrm{m}$ was placed in a $100-\mathrm{keV} \bar{p}$ beam. The setup is shown in Fig. 4(a). Annihilation events were recorded with a high-resolution photographic emulsion for $6.5 \mathrm{~h}$. The reference position (without deflection) was determined by allowing part of the $\bar{p}$ beam to reach the detector via a grating placed in direct contact with it. Furthermore, both the deflectometer and the contact grating were illuminated with diffuse light, for which the material grating acted like a Talbot-Lau interferometer [33]. 
Since photons travel at the speed of light, their deflection during the short distance traveled is negligible. Their maxima in the plane of the detector agree with those of the antiprotons from the contact grating, as shown in the left graph of Fig. 4(b). On the other hand, antiprotons passing through the deflectometer are displaced in the positive $y$ direction (right pane). The graph in Fig. 4(c) shows the locations of $\bar{p}$ annihilations modulo the grating period (blue bins and black line) compared to the light signal (red line). Quantitatively, a vertical deflection of $\delta y=9.8 \pm 0.9$ (stat) \pm 6.4 (syst) $\mu \mathrm{m}$ was observed, corresponding to an upward force of $530 \pm 50$ (stat) \pm 350 (syst) aN [32]. This result is compatible with the Lorentz force due to a magnetic fringe field of about $8 \mathrm{G}$. While the force of gravity acting on $\overline{\mathrm{H}}$ atoms is many orders of magnitude smaller, their expected deflection is comparable due to the significantly lower velocity and longer flight distance in the full-scale experiment.

\subsection{Positronium lifetime spectroscopy}

The direct excitation of Ps to a Rydberg level is challenging due to the unavailability of a suitable laser. A scheme using $n=2$ as intermediate state, on the other hand, has been demonstrated [34, 35]. We are pursuing an alternative path via the $n=3$ level, which requires a lower laser power to reach saturation of the $n=3 \longrightarrow$ Rydberg transition due to the longer lifetime of the intermediate state. The power dissipated in the cryogenic environment negatively affects both the production efficiency and the temperature of the formed $\overline{\mathrm{H}}$ and must therefore be kept to a minimum. As a prerequisite for the use of the novel excitation scheme, we studied the structure of Ps by single-shot positronium annihilation lifetime spectroscopy (SSPALS) [36]. The technique is based on monitoring the lifetime of Ps, which changes as a function of its internal quantum state. Both the excitation of Ps to a Rydberg state and two-step photodetachment were demonstrated [37].

In the setup, which is shown in Fig. 5(a), Ps was produced by directing a $3.3 \mathrm{keV}$ beam of $e^{+}$from a custom-built bunching and electrostatic transport system [38] onto a high-efficiency converter target. Both the ultraviolet (UV) laser with $\lambda_{\mathrm{UV}} \approx 204 \ldots 206 \mathrm{~nm}$ and the infrared laser with $\lambda_{\mathrm{IR}} \approx 1064 \mathrm{~nm}$ were directed onto the target region. Ps annihilations were recorded with a lead tungstate scintillator coupled to a photomultiplier placed $40 \mathrm{~mm}$ above the converter. In a first experiment, the IR laser was tuned to a transition from $n=3$ to the continuum, dissociating the Ps. The free positrons were drawn away from the detector, resulting in an overall decrease of the observed annihilations, as shown by the grey line in the left pane of Fig. 5(b). The UV laser was then scanned around the $n=1 \longrightarrow 3$ and the relative change in the number of annihilations recorded as a function of $\lambda_{\mathrm{UV}}$. The resulting resonance (right) yields a wavelength of 205.05(2) nm [37], in good agreement with the theoretical value.

In a second measurement, the IR laser was tuned to a transition from $n=3$ to a Rydberg state while the UV laser wavelength was kept constant on the $n=3$ resonance. The Rydberg excitation increases the Ps lifetime because the overlap of the $e^{+}$and $e^{-}$wavefunctions is reduced. The SSPALS data thus show a reduction of annihilations up to $300 \mathrm{~ns}$ after Ps production, followed by an excess in the time window $300 \ldots 600 \mathrm{~ns}$ when Ps hit the chamber wall, as illustrated in the left pane of Fig. 5(c). The relative change in the number of annihilations at later times is shown in the right pane. Resonances of the $n=15,16,17$ are clearly visible [37] and in good agreement with prior spectroscopic surveys. For higher Rydberg states, different $n$ manifolds start to overlap in a continuum of energy levels. These measurements thus demonstrate the first laser excitation of Ps to the $n=3$ state, enhancing the overall Ps excitation efficiency and reducing the laser energy deposited in the target region.

\section{Conclusions and outlook}

While the foundations of general relativity are well-tested with ordinary matter, the gravitational interaction of antimatter has never been studied experimentally. In a quantum theory of gravity, additional 

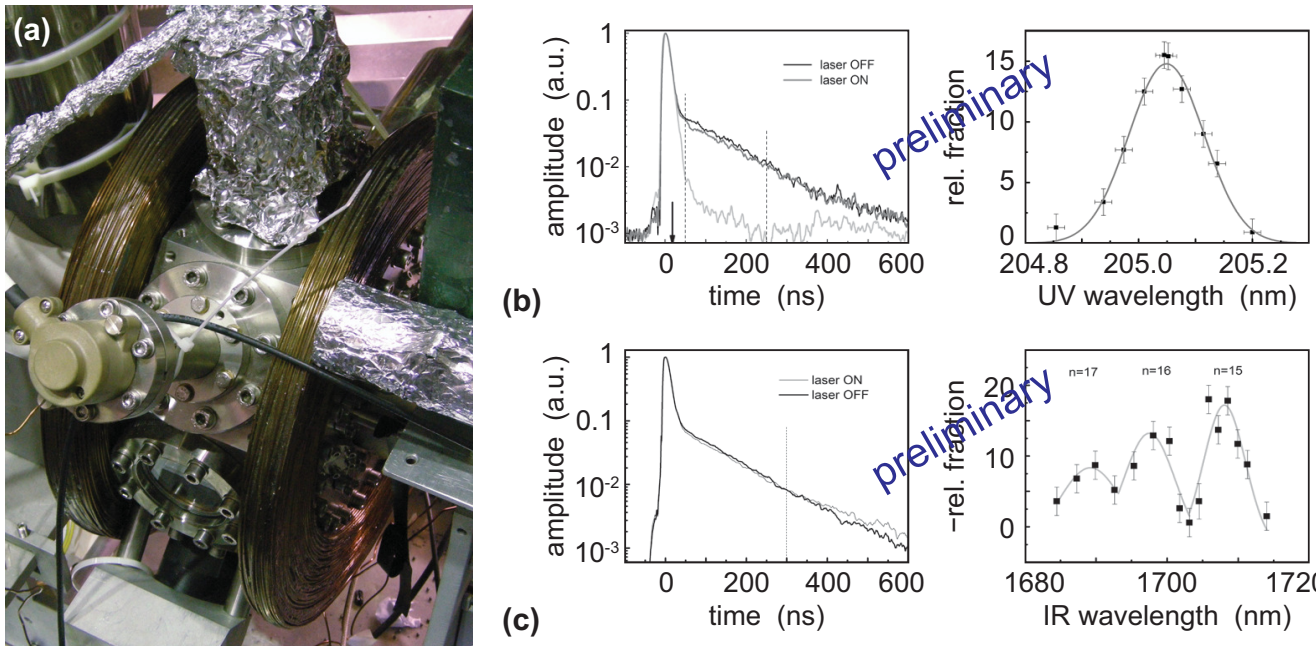

\section{(b)}

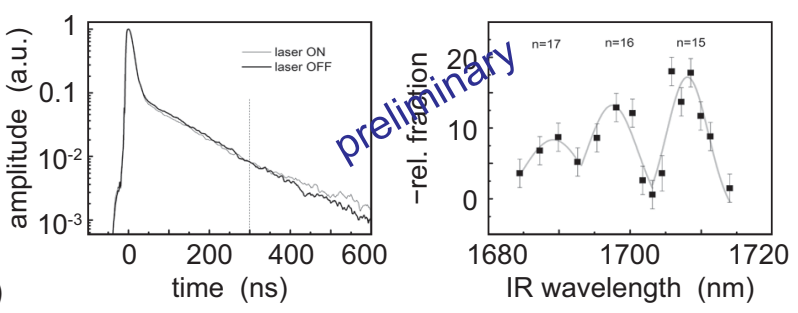

Figure 5. (a) Vacuum chamber which houses the positron annihilation lifetime spectroscopy setup. Positrons enter from the right and impinge on a central target. Lasers enter from the front; an annihilation detector is placed $40 \mathrm{~mm}$ above the target. (b) SSPALS data for the IR laser tuned to a transition from $n=3$ to the continuum (left) and the resulting resonance of the $n=1 \longrightarrow 3$ transition (right). (c) SSPALS data for the IR laser tuned to $n=3 \longrightarrow$ Rydberg (left) and observed resonances for $n=3 \longrightarrow 15,16,17$ (right) (from Ref. [37]).

exchange particles may lead to an anomalous force between matter and antimatter particles. The AEGIS experiment is gearing up to conduct the first measurement of the gravitational acceleration of an antimatter particle, by deflectometry or interferometry with a slow $\overline{\mathrm{H}}$ beam. A large part of the apparatus has been completed. Both $\bar{p}$ and $e^{+}$have been captured and stored for extended periods in the AEGIS ion traps. The ability to detect the deflection of an antimatter particle beam by a few $\mu \mathrm{m}$ has been demonstrated. The excitation of Ps to Rydberg states via the $n=3$ level has been achieved, paving the way for the efficient production of $\overline{\mathrm{H}}$ at low temperature. A measurement of antimatter gravity at the level of $1 \%$ is therefore within reach in the medium term.

\section{Acknowledgements}

This work was supported by the European Research Council, the European Social Fund, the Austrian Ministry for Science, Research and Economy, the German Research Foundation, the Istituto Nazionale di Fisica Nucleare, the Research Council of Norway, the Bergen Research Foundation, the Russian Ministry of Education and Science, the Russian Academy of Sciences and the John Templeton Foundation.

\section{References}

[1] J.Y. Hémery, S. Maury, Nucl. Phys. A 655, 345c (1999), doi : 10.1016/S0375-9474(99) Q0223-7

[2] W. Pauli, in Niels Bohr and the Development of Physics, edited by W. Pauli, L. Rosenfeld, V. Weisskopf (Pergamon Press, New York, 1955), p. 30

[3] A.D. Sakharov, JETP Lett. 5, 24 (1967), http://www.jetpletters.ac.ru/ps/1643/ article_25089.shtml 
[4] O. Bertolami, D. Colladay, V.A. Kostelecký, R. Potting, Phys. Lett. B 395, 178 (1997), doi : 10. 1016/S0370-2693(97)00062-2

[5] T. Goldman, R.J. Hughes, M.M. Nieto, in First Workshop on Antimatter Physics at Low Energy (Fermilab, Batavia, IL, 1986), p. 185, http://lss . fnal.gov/conf/C860410/p185.pdf

[6] T.A. Wagner, S. Schlamminger, J.H. Gundlach, E.G. Adelberger, Class. Quantum Grav. 29, 184002 (2012), doi : $10.1088 / 0264-9381 / 29 / 18 / 184002$

[7] M.M. Nieto, T. Goldman, Phys. Rep. 205, 221 (1991), doi:10.1016/0370-1573(91) 90138-C

[8] P. Morrison, Am. J. Phys. 26, 358 (1958), doi : 10. 1119/1. 1996159

[9] M. Fischler, J. Lykken, T. Roberts, Fermilab Report FN-0822-CD-T, Fermilab, Batavia, IL (2008), http://lss . fnal.gov/archive/test-fn/0000/fermilab-fn-0822-cd-t.pdf

[10] S. Schlamminger et al., Phys. Rev. Lett. 100, 041101 (2008), doi : 10.1103/PhysRevLett. 100.041101

[11] T.W. Darling, F. Rossi, G.I. Opat, G.F. Moorhead, Rev. Mod. Phys. 64, 237 (1992), doi : 10. 1103/RevModPhys. 64.237

[12] M. Amoretti et al. (ATHENA Collaboration), Nature 419, 456 (2002), doi:10.1038/ nature01096

[13] G. Gabrielse, S.L. Rolston, L. Haarsma, W. Kells, Phys. Lett. A 129, 38 (1988), doi : 10. 1016/ 0375-9601(88) 90470-7

[14] G. Drobychev et al. (AEGIS Proto-Collaboration), Proposal to the SPS Experiments Committee SPSC-P-334, CERN, Geneva (2007), http: //cds . cern. ch/record/1037532

[15] A. Kellerbauer et al. (AEGIS Proto-Collaboration), Nucl. Instrum. Methods B 266, 351 (2008), doi : 10.1016/j .nimb.2007.12.010

[16] M. Doser et al. (AEGIS Collaboration), Class. Quantum Grav. 29, 184009 (2012), doi : 10 . 1088/0264-9381/29/18/184009

[17] M. Amoretti et al. (ATHENA Collaboration), Phys. Lett. B 578, 23 (2004), doi : 10. 1016/j . physletb.2003.10.062

[18] C.H. Storry et al. (ATRAP Collaboration), Phys. Rev. Lett. 93, 263401 (2004), doi : 10.1103/ PhysRevLett. 93.263401

[19] A. Kellerbauer, J. Walz, New J. Phys. 8, 45 (2006), doi : 10. 1088/1367-2630/8/3/045

[20] E. Jordan et al., Phys. Rev. Lett. 115, 113001 (2015), doi:10.1103/PhysRevLett. 115. 113001

[21] P. Yzombard et al., Phys. Rev. Lett. 114, 213001 (2015), doi : 10.1103/PhysRevLett. 114. 213001

[22] D.W. Gidley, H.G. Peng, R.S. Vallery, Annu. Rev. Mater. Res. 36, 49 (2006), doi : 10.1146/ annurev.matsci.36.111904.135144

[23] G. Consolati et al., Chem. Soc. Rev. 42, 3821 (2013), doi : 10.1039/c2cs35454c

[24] C.G. Fischer et al., Phys. Rev. B 71, 180102 (2005), doi : 10.1103/10.1103/PhysRevB. 71. 180102

[25] F. Castelli, I. Boscolo, S. Cialdi, M.G. Giammarchi, Phys. Rev. A 78, 052512 (2008), doi : 10.1103/PhysRevA.78.052512

[26] E. Vliegen, S.D. Hogan, H. Schmutz, F. Merkt, Phys. Rev. A 76, 023405 (2007), doi : 10. 1103 / PhysRevA.76.023405

[27] M.K. Oberthaler et al., Phys. Rev. A 54, 3165 (1996), doi : 10.1103/PhysRevA . 54.3165 
[28] S. Aghion et al. (AEGIS Collaboration), JINST 8, P08013 (2013), doi : 10 . 1088/1748-0221/ $8 / 08 / \mathrm{P} 08013$

[29] S. Aghion et al. (AEGIS Collaboration), JINST 9, P06020 (2014), doi : 10 . 1088/1748-0221/ 9/06/P06020

[30] J. Storey et al. (AEGIS Collaboration), JINST 10, C02023 (2015), doi : 10 . 1088/1748-0221/ $10 / 02 / C Q 2023$

[31] S. Cialdi et al., Nucl. Instrum. Methods B 13, 1527 (2011), doi : 10.1016/j .nimb. 2011.04. 108

[32] S. Aghion et al. (AEGIS Collaboration), Nature Commun. 5, 4538 (2014), doi: 10.1038/ ncomms 5538

[33] E. Lau, Ann. Physik 437, 417 (1948), doi : 10. 1002/andp. 19484370709

[34] D.B. Cassidy, T.H. Hisakado, H.W.K. Tom, A.P. Mills, Phys. Rev. Lett. 108, 043401 (2012), doi : 10.1103/PhysRevLett. 108.043401

[35] T.E. Wall et al., Phys. Rev. Lett. 114, 173001 (2015), doi:10.1103/PhysRevLett.114. 173001

[36] D.B. Cassidy, A.P. Mills, Nucl. Instrum. Methods A 580, 1338 (2007), doi : 10. 1016/j .nima. 2007.06 .078

[37] S. Aghion et al. (AEGIS Collaboration), Phys. Rev. A (submitted 2015-09-25)

[38] S. Aghion et al. (AEGIS Collaboration), Nucl. Instrum. Methods B 362, 86 (2015), doi : 10. $1016 /$ j.nimb.2015.08.097 